\title{
Only $10 \%$ of people with schizophrenia show sustained improvements over 3 years
}

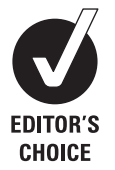

\section{QUESTION}

Question: What proportion of people with schizophrenia have favourable long-term outcomes, and which factors predict a more favourable outcome?

Population: 2327 adults treated for schizoaffective disorders or schizophrenia (DSM-IV) and participating in the United States Schizophrenia Care and Assessment Program (US-SCAP). The majority of participants were outpatients $(93.5 \%)$.

Setting: Community mental health centres, community and state hospitals, university healthcare systems, and Department of Veterans Affairs Health Services, USA; from July 1997 to September 2003.

Prognostic factors: Sixty-two factors were assessed as possible prognostic variables, including patient-reported variables, clinician-rated variables and medical record based resource utilisation. Symptom severity was assessed at baseline and annually using the Positive and Negative Symptom Scale score. Acute healthcare utilisation (hospitalisation, emergency services) was assessed using medical records. Quality of life and functional outcomes (eg, volunteering, working for pay, attending school, caring for children and keeping house) were assessed (SCAP Health questionnaire, Global Assessment of Functioning, Quality of Life Scales) at enrolment and every 6 months. Depressive symptoms were assessed at enrolment and annually (Montgomery-Asberg Depression Rating Scale). Medication adherence and medication effects, including adverse events (Simpson-Angus Scale) and tardive dyskinesia (Abnormal Involuntary Movement Scale) were assessed annually. Socio-demographic data were collected at baseline.

Outcomes: Sustained favourable long-term outcome. The health state of each participant, determined by hierarchical cluster analysis of symptom severity, level of functioning and use of acute care services, was identified at baseline, and annually thereafter. Participants were grouped into best, second best, middle, second worst, or worst health state clusters. Changes in health state cluster from year 1 to year 2 , and year 2 to year 3 were determined. Sustained favourable long-term outcome was defined as being in the best health state cluster for 2 years postbaseline.

\section{METHODS}

Design: Retrospective analysis of data from a prospective observational study.

Follow-up period: 3 years.

\section{MAIN RESULTS}

Data from 1942 (83\%) of participants were available for cluster analysis. At baseline, 503 participants were in the best health state cluster (25.9\%), 992 in the second best (51.1\%), 145 in the middle $(7.47 \%), 53$ in the second worst $(2.73 \%)$ and $249(12.8 \%)$ in the worst. Postbaseline data were available for 1635 participants $(70 \%)$. At year 1, 269 participants were closest to best health state cluster (23\%). From year 1 to year 2, 209 (13\%) were closest to best health state cluster. A sustained favourable longterm outcome was achieved by 157 participants (10\%). Over 3 years, $85 \%(\mathrm{n}=688)$ did not have a sustained shift in health state, $10 \%(n=84)$ had improved and $4 \%(n=34)$ had worsened health states. Nine baseline variables were significantly associated with sustained favourable long-term outcome. The likelihood of a sustained favourable long-term outcome was associated with being employed (OR 1.98, 95\% CI 1.34 to 2.91); ability to shop independently (OR 1.76, 95\% CI 1.19 to 2.59 ); ability to undertake independent leisure activities (OR 1.75, 95\% CI 1.10 to 2.79); experiencing clearer thoughts from medications (OR 1.21, 95\% CI 1.04 to 1.40 ); better quality of life (OR $1.64,95 \%$ CI 1.32 to 2.03); better global functioning (OR 1.04, 95\% CI 1.02 to 1.06 ); and more daily activities (OR $1.27,95 \%$ CI 1.06 to 1.52 ). The likelihood of experiencing a sustained favourable long-term outcome was lower in those who had received individual therapy (OR $0.47,95 \%$ CI 0.25 to 0.88 ), or who had been a victim of crime (OR $0.38,95 \%$ CI 0.17 to 0.85 ).

\section{CONCLUSIONS}

Most people with schizophrenia in naturalistic care settings have no sustained change in health status over 3 years. Better functioning on certain measures at baseline predicted sustained favourable outcome over this period.

\section{ABSTRACTED FROM \\ Cuyún Carter GB, Milton DR, Ascher-Svanum H, et al. Sustained favorable long-term outcome in the treatment of schizophrenia: a 3-year prospective observational study. BMC Psychiatry 2011;11:143}

Correspondence to: Eli Lilly and Company, Global Health Outcomes, Indianapolis, IN 46285, USA; cuyun_carter_gebra@lilly.com

Sources of funding: Eli Lilly and Company.

- Notes and references are available online only at http://ebmh.bmj.com
C uyún-Carter et al report the findings of a 3 year extensive non-randomised study on patients with schizophrenia spectrum disorders treated in routine clinical practice. A major objective of this study was to report on the treatment outcome and respective predictors. Apart from the large study sample, the most impressive issue is the multi-dimensional definition of outcome including symptoms, functioning and care utilisation. Based on this criteria, only $10 \%$ achieved a favourable and sustained outcome, which is somewhat lower than generally found in schizophrenia cases. ${ }^{1}$ Of various assessments, many baseline characteristics emerged as significant predictors which can be summarised as 'favourable baseline characteristics predict favourable outcome'; a result representative for schizophrenia. In multiple logistic regression analysis, nine predictors remained significant and the authors conclude that this might enable individual treatment decisions. Unfortunately, many different predictors have been identified in several studies. However, so far, there are no set of valid predictors which enables individual prognosis. ${ }^{2}$ Among others, this is due to the approach of a multiple regression analysis which identifies predictors which best represent the analysed sample, however, this result cannot be generalised to other samples.

Given the large sample in the present study, it would have been reasonable to primarily split and cross-validate the set of predictors identified in both samples separately. In addition, identifying individuals requires distinct criteria and cut-off points which do not seem feasible based on the study results (just small differences in the means between the favourable vs not favourable patients). Furthermore, the authors interpret predictors causally by concluding that training in role and occupational functioning might lead to favourable outcome not considering that this association might be caused by a third (moderator) variable (eg, neurophysiological factors which can hardly be affected by psychosocial treatment). Thus, the result of this comprehensive study in real-world clinical setting contributes data to the objective of individual prognosis and treatment decisions; however, more research is needed in this field.

\section{Wolfgang Gaebel, Mathias Riesbeck}

Department of Psychiatry and Psychotherapy, LVR Klinikum Düsseldorf, Medical Faculty, Heinrich Heine University Düsseldorf, Düsseldorf, Germany

Competing interests WG has received symposia support from Janssen-Cilag, Neuss/Germany, and Lilly Deutschland, Bad Homburg/Germany and is a member of the advisory board of Lundbeck International Neuroscience Foundation (LINF), Denmark. MR has no conflicting interests. 